\title{
Telephone-based postoperative surveillance protocol for hepatobiliary cancer during the COVID-19 outbreak
}

\author{
Nadia Russolillo $^{1}$ (D) Elisa Sperti ${ }^{2} \cdot$ Alessandro Ferrero $^{1}$
}

Received: 1 June 2020 / Accepted: 3 June 2020 / Published online: 10 June 2020

(C) Italian Society of Surgery (SIC) 2020

Routine oncological follow-up is provided for the detection of locoregional or distant relapse, the provision of psychosocial support and the detection and management of side effects of therapy [1].

Especially in this potentially curative setting, delay of surveillance activities should be avoided, especially for highrisk patients [2]. Soon after the beginning of the Covid-19 emergency, Italian Association of Medical Oncology recommended to replace traditional visits with phone calls/online exchange of clinical documentation [3]. In our opinion, the hospital system must maintain the continuity of care as much as possible, adapting the operating procedures to the Covid-19 emergency. Our hospital is a tertiary hepato-pancreato-biliary center, and approximately 180 hepatectomies are performed and over 350 patients are managed per year. Postoperative surveillance for liver tumors is held in an outpatients department managed by surgeons and oncologists twice a week (almost 10 patients per day). Our hospital has been actively involved in the management of the pandemic emergency with many divisions converted to COVID divisions. As a result, more than 500 patients have been hospitalized for COVID-19 in the last 3 in months.

To reduce the number of hospital accesses during the emergency, we developed a telephone-based surveillance protocol.

Follow-up by telephone has been tried in other cancer settings, with good outcomes in terms of detecting medical problems and providing support to oncologic patients [4].

According to our protocol, telephone-based follow-up is managed by a surgeon or an oncologist.

The content of each call is structured using a standardized checklist covering 5 areas:

Nadia Russolillo

nrussolillo@mauriziano.it

1 Department of General and Oncological Surgery,

Mauriziano Hospital, Turin, Italy

2 Department of Oncology, Mauriziano Hospital, Turin, Italy
1. General health (e.g. mobility, energy level fatigue, appetite/dietary intake, weight status, ability to care for self/ necessary assistance).

2. Bowel function (e.g. continence, stoma management).

3. Disease-specific quality of life.

4. Check of blood tests results including tumor markers (blood cell count, $\mathrm{Na}, \mathrm{K}$, creatinine, total bilirubin, direct bilirubin, ALT, GGT, alkaline phosphatase, albumin, INR, tumor markers CEA, Ca19.9 or AFP).

5. Check of abdominal imaging reports (ultrasonography or thoracoabdominal CT/abdominal MRI).

Patients are invited to send the results via email or post. In situations where further exams are warranted, the clinicians arrange appointments as appropriate. Timing and type of the subsequent oncological surveillance is based on our standard protocol [5]. Patients are asked to perform blood tests and imaging close to home and in outpatients imaging centers rather than in hospital and university radiology departments currently involved in the Covid-19 emergency.

Clinical findings are detailed on a standard report in the patient's hospital file, specifying that is recorded in remote modality. Finally, a copy of the medical report, along with prescriptions needed for the subsequent appointment is sent to the patient by email or by post.

In a period of 2 months telephone-based postoperative surveillance were performed in 84 patients. Liver recurrence was detected by follow-up image examination in 2 cases. Further exams were scheduled for 3 patients with elevation of tumor markers. All but two patients were able to send and receive the documentation by email. Of course, situation is dynamic, and policies and recommendations may change at any time. Our experience confirms that, nowadays, telephone consultation is an acceptable type of healthcare provision to support patients until the epidemic slows its pace. 
Acknowledgement We thanks Dr. Serena Langella and Prof. Massimo Di Maio who actively participated to the the follow-up protocol development.

Funding The authors received no specific funding for this work.

\section{Compliance with ethical standards}

Conflict of interest The authors confirm that there are no known conflicts of interest associated with this publication.

Ethical approval All the authors made a significant contribution to the work and they reviewed and agreed the final version of the article before submission.

Informed consent Informed consent is not necessary for this type of study.

\section{References}

1. Furman MJ, Lambert LA, Sullivan ME, Whalen GF (2013) Rational follow-up after curative cancer resection. J Clin Oncol $31: 1130-1133$
2. van Meer S, de Man RA, Coenraad MJ, Sprengers D, van Nieuwkerk KM, Klümpen HJ et al (2015) Surveillance for hepatocellular carcinoma is associated with increased survival: results from a large cohort in the Netherlands. J Hepatol 63:1156-1163

3. Lambertini M, Toss A, Passaro A, Criscitiello C, Cremolini C, Cardone C, Loupakis F, Viscardi G, Meattini I, Dieci MV, Ferrara R, Giusti R, Maio MD (2020) Cancer care during the spread of coronavirus disease 2019 (COVID-19) in Italy: young oncologists' perspective. ESMO Open 5(2):e000759

4. Dickinson R, Hall S, Sinclair JE, Bond C, Murchie P (2014) Using technology to deliver cancer follow-up: a systematic review. BMC Cancer 14:311

5. Russolillo N, Sperti E, Langella S, Menonna F, Allieta A, Di Maio M, Ferrero A (2020) Impact of primary tumor location on patterns of recurrence and survival of patients undergoing resection of liver metastases from colon cancer. HPB (Oxford) 22:116-123

Publisher's Note Springer Nature remains neutral with regard to jurisdictional claims in published maps and institutional affiliations. 